\title{
MORE ON REAL-VALUED MEASURABLE CARDINALS AND FORCING WITH IDEALS
}

by

\author{
Moti Gitik \\ School of Mathematical Sciences \\ Sackler Faculty of Exact Sciences \\ Tel Aviv University \\ Ramat Aviv 69978 Israel
}

\author{
Saharon Shelah \\ Hebrew University of Jerusalem \\ Department of Mathematics \\ Givat Ram, Jerusalem
}

\begin{abstract}
.
(1) It is shown that if $c$ is real-valued measurable then the Maharam type of $(c, \mathcal{P}(c), \sigma)$ is $2^{c}$. This answers a question of D. Fremlin $[\mathrm{Fr},(\mathrm{P} 2 \mathrm{f})]$.

(2) A different construction of a model with a real-valued measurable cardinal is given from that of R. Solovay [So]. This answers a question of D. Fremlin $[\mathrm{Fr},(\mathrm{P} 1)]$.

(3) The forcing with a $\kappa$-complete ideal over a set $X,|X| \geq \kappa$ cannot be isomorphic to Random $\times$ Cohen or Cohen $\times$ Random. The result for $X=\kappa$ was proved in [Gi-Sh1] but as was pointed out to us by M. Burke the application of it in [Gi-Sh2] requires dealing with any $X$.
\end{abstract}


In Section 1 we deal with the Maharam types of real-valued measurable cardinals. The result (1) stated in the abstract and its stronger version are proved. The proofs are based on Shelah's strong covering lemmas and his revised power set operation.

In Section 2 a model with a real-valued measurable which is not obtained as the Solovay one by forcing random reals over a model with a measurable.

In Section 3, the result (3) stated in the abstract is proved.

Theorem 1.1 and the construction of Section 2 is due to the first author. Theorem 1.2 is joint and the result of Section 3 is due to the second author.

We are grateful to David Fremlin for bringing the questions on real-valued measurability to our attention. His excellent survey article [Fr] gave the inspiration for the present paper. We wish to thank the Max Burke for pointing out a missing stage in the argument of $[\mathrm{Gi}-\mathrm{Sh} 2]$.

\section{On Number of Cohen or Random Reals}

D. Fremlin asked the following in $[\mathrm{Fr},(\mathrm{P} 2 \mathrm{f})]$ :

If $c$ is a real-valued measurable with witnessing probability $\nu$, does it follow that the Maharam type of $(c, \mathcal{P}(c), \nu)$ is $2^{c}$ ? or in equivalent formulation:

If $c$ is a real-valued measurable does the forcing with witnessing ideal isomorphic to the forcing for adding $2^{c}$ random reals?

The next theorem provides the affirmative answer.

Theorem 1.1. Suppose that $I$ is a $2^{\aleph_{0}}$-complete ideal over $2^{\aleph_{0}}$ and the forcing with it (i.e. $\mathcal{P}\left(2^{\aleph_{0}}\right) / I$ ) is isomorphic to the adding of $\lambda$-Cohen or $\lambda$-random reals. Then $\lambda=2^{2^{\aleph_{0}}}$.

Proof: Suppose otherwise. Denote $2^{\aleph_{0}}$ by $\kappa$. Let $j: V \rightarrow N$ be a generic elementary embedding.

Claim 1. $j(\kappa)>\left(\lambda^{+}\right)^{V}$.

Proof: By a theorem of Prikry [Pr] (see also [Gi-Sh2] for a generalization) for every $\tau<\kappa 2^{\tau}=2^{\aleph_{0}}=\kappa$. Then, in $N, 2^{\kappa}=j(\kappa)$. But $(\mathcal{P}(\kappa))^{V} \subseteq N$, so $j(\kappa) \geq\left(2^{\kappa}\right)^{V}$. By [Gi-Sh2], then $\left(2^{\kappa}\right)^{V}=\operatorname{cov}\left(\lambda, \kappa, \aleph_{1}, 2\right.$. So $\operatorname{cov}\left(\lambda, \kappa, \aleph_{1}, 2\right) \geq \lambda^{+}$. Clearly,

$$
\operatorname{cov}\left(\lambda, \kappa, \aleph_{1}, 2\right) \leq \operatorname{cov}\left(\lambda, \aleph_{1}, \aleph_{1}, 2\right) \leq\left(\operatorname{cov}\left(\lambda, \aleph_{1}, \aleph_{1}, 2\right)\right)^{N}
$$


The last inequality holds since $N$ is obtained by a c.c.c. forcing and so every countable set of ordinals in $N$ can be covered by a countable set of $V$. By Shelah [Sh430], in $N$ $\operatorname{cov}\left(\lambda, \aleph_{1}, \aleph_{1}, 2\right)<j(\kappa)$. Hence $\lambda^{+} \leq \operatorname{cov}\left(\lambda, \kappa, \aleph_{1}, 2\right) \leq\left(\operatorname{cov}\left(\lambda, \aleph_{1}, \aleph_{2}\right)\right)^{N}<j(\kappa)$.

of the claim.

By Shelah [Sh 430] there is $S \subseteq[\kappa]]^{\leq \aleph_{0}}$ unbounded of cardinality $\kappa$. For $\alpha<\kappa$ let $S\left\lceil\alpha=\{P \in S \mid P \subseteq \alpha\}\right.$. Fix a function $f \in^{\kappa} \kappa$ representing $\kappa$ is a generic ultrapower and restrict everything to a condition forcing this.

Claim 2. $\left\{\alpha<\kappa|S| f(\alpha)\right.$ is not unbounded in $\left.[f(\alpha)]^{\leq \aleph_{0}}\right\} \in I$.

Proof: Otherwise, in a generic ultrapower $N \cdot j(S) \mid \kappa=S$ will be bounded. I.e. there will be some $t \subseteq \kappa$ countable such that for every $s \in S s \nsupseteq t$. Using c.c.c. of the forcing we find a countable subset of $\kappa$ in $V, t^{*} \supseteq t$. Since $S$ is unbounded in $V$ some $s \in S$ contains $t^{*}$. Contradiction. of the claim.

Let $N$ be a generic ultrapower. By [Gi-Sh1] there are in $N$ at least $\kappa$ Cohen (or random) reals over $V$.

Claim 3. There exists a sequence $\left\langle r_{\alpha} \mid \alpha<\kappa\right\rangle$ of reals in $V$ so that

(1) every real of $V$ appears in $\left\langle r_{\alpha} \mid \alpha<\kappa\right\rangle$.

(2) for almost all $\alpha(\bmod I)\left\langle r_{\alpha+i} \mid i<f(\alpha)\right\rangle$ are $f(\alpha)$-Cohen (random) generic over $L\left[S \mid f(\alpha),\left\langle r_{\beta} \mid \beta<f(\alpha)\right\rangle\right]$.

Proof: Construct $\left\langle r_{\alpha} \mid \alpha<\kappa\right\rangle$ by induction. On nonlimit stages add reals in order to satisfy (1). For limit $\alpha^{\prime} s$ with $S \uparrow f(\alpha)$ unbounded in $[f(\alpha)]^{\leq \aleph_{0}}$, add $f(\alpha)$-Cohen (or random) reals. It is possible since there are at least $\kappa$ candidates in a generic ultrapower by [Gi-Sh1].

$\square$ of the claim.

Now work in $N . r n g f\left\lceil A\right.$ is unbounded in $\kappa$, for every $A \notin I$. Let $j\left(\left\langle r_{\alpha} \mid \alpha<\kappa\right\rangle\right)=$ $\left\langle r_{\alpha} \mid \alpha<j(\kappa)\right\rangle$ where $\left\langle r_{\alpha} \mid \alpha<\kappa\right\rangle$ is a sequence given by Claim 3.

Then, using Claim 3 in $N$ we can find some $\alpha^{*}<j(\kappa)$ satisfying (2) of Claim 3 such that $j(S)\left\lceil\alpha^{*}\right.$ is unbounded in $\left[\alpha^{*}\right]^{\leq \aleph_{0}}$ and $j(f)\left(\alpha^{*}\right) \geq\left(\lambda^{+}\right)^{V}$. It is possible since by Claim $1,\left(\lambda^{+}\right)^{V}<j(\kappa)$ and, in $V$ the range of $f$ restricted to a set not in $I$ is unbounded in $\kappa$.

The following will provide the contradiction and complete the proof of the theorem. 
Claim 4. $\left\langle r_{\alpha^{*}+i} \mid i<j(f)\left(\alpha^{*}\right)\right\rangle$ is a sequence of Cohen (random) reals over $V$.

Proof: $\left\langle r_{\alpha^{*}+i} \mid i<j(f)\left(\alpha^{*}\right)\right\rangle$ is Cohen (random)-generic over $L\left[j(S)\left\lceil j(f)\left(\alpha^{*}\right),\left\langle r_{\beta}\right|\right.\right.$ $\left.\left.\beta<j(f)\left(\alpha^{*}\right)\right\rangle\right]$. In particular, also over $L\left[j(S)\left\lceil j(f)\left(\alpha^{*}\right),\left\langle r_{\beta} \mid \beta<\kappa\right\rangle\right]\right.$. But $\left\langle r_{\beta} \mid \beta<\kappa\right\rangle$ is a list of all the reals of $V$. It is enough to show that $j(S)\left\lceil j(f)\left(\alpha^{*}\right) \cap\left(\left[j(f)\left(\alpha^{*}\right)\right]^{<\aleph_{0}}\right)^{V}\right.$ is unbounded.

We note that the very strong covering holds between $L\left[S\left\langle r_{\alpha} \mid \alpha<\kappa\right\rangle\right]$ and $L\left[j(S),\left\langle r_{\alpha}\right|\right.$ $\alpha<\kappa\rangle]$. Also, for any $b \in V \cap H_{\kappa}$, the same is true about $\left.\left.L\left[b, S,\left\langle r_{\alpha}\right| \alpha<\kappa\right]\right\rangle\right]$ and $L\left[b, j(S),\left\langle r_{\alpha} \mid \alpha<\kappa\right\rangle\right]$. Then the same holds in $N$, between

$$
L\left[b, j(S)\left\lceil j(f)\left(\alpha^{*}\right),\left\langle r_{\beta} \mid \beta<j(f)\left(\alpha^{*}\right)\right\rangle\right] \text { and } L\left[b, j(S),\left\langle r_{\beta} \mid \beta<j(\kappa)\right\rangle\right]\right. \text {, }
$$

for every $b \in V \cap H_{\kappa}$. Now consider $V, V[G]$. Using c.c.c. it is easy to find the winning strategy for $V$. Take a play which is according such a strategy and of the length $<\kappa$, in sense of $[\operatorname{Sh} 420,2.6, \mu(3)]$. Then it belongs to $L\left[j(S),\left\langle r_{\beta} \mid \beta<j(\kappa)\right\rangle\right]$ since the last model agrees with $V[G]$ about small sequences. Now use the Shelah Strong Covering [Sh580] for $L\left[j(S)\left\lceil j(f)\left(\alpha^{*}\right),\left\langle r_{\beta}\right| \beta<j(f)\left(\alpha^{*}\right)\right], V\right.$ and $V[G]$. We will obtain

$$
P \in V \cap L\left[j(S)\left\lceil j(f)\left(\alpha^{*}\right),\left\langle r_{\beta}\right| \beta<j(f)\left(\alpha^{*}\right)\right] \cap\left[j(f)\left(\alpha^{*}\right)\right]^{\leq \aleph_{0}} .\right.
$$

Since $|P|=\aleph_{0}$ all its subsets in $V$ are also in $L\left[S,\left\langle r_{\alpha} \mid \alpha<\kappa\right\rangle\right] \subseteq L\left[j(S)\left\lceil j(f)\left(\alpha^{*}\right),\left\langle r_{\beta}\right|\right.\right.$ $\left.\left.\beta<j(f)\left(\alpha^{*}\right)\right\rangle\right]$. Hence the Cohen (random) genericity over the last model is equivalent the Cohen (random) genericity over $V$.

Let us now prove a stronger statement which relies on a different property.

Theorem 1.2. Suppose that $I$ is a $\kappa$-complete ideal over $\kappa$ and the forcing with it (i.e. $\mathcal{P}(\kappa) / I)$ is isomorphic to the forcing for adding $\lambda$-Cohen or $\lambda$-random reals. Assume also $\left(^{*}\right)$ some condition forces that " $j(\kappa) \geq\left(2^{\kappa}\right)^{V}$ ", where $j$ is a generic embedding. Then $\lambda>2^{<\kappa}$ implies $\lambda=2^{\kappa}$.

Proof: Without loss of generality, let us assume that the weakest condition, i.e. $\kappa$ forces $\left.{ }^{*}\right)$. Suppose that $\lambda<2^{\kappa}$. Then $\lambda<j(\kappa)$. By [Sh430], then in a generic ultrapower $N$ $\operatorname{cov}\left(\lambda, \aleph_{1}, \aleph_{1}, 2\right)<j(\kappa)$. However, in $\left.V \lambda^{+} \leq \operatorname{cov}\left(\lambda, \kappa, \aleph_{1}, 2\right) \leq \operatorname{cov}\left(\lambda, \aleph_{1} \aleph_{1}, 2\right)\right)^{N}<j(\kappa)$. The first inequality holds by [Gi-Sh2]. Hence $\lambda^{+}<j(\kappa)$. 
Now, by [Sh 430, 460, 2.6], there are regular $\delta<\mu<\kappa$ such that $\operatorname{cov}(\lambda, \mu, \mu, \delta)=\lambda$.

Let us assume for simplification of the notation that $\mu=\aleph_{2}, \delta=\aleph_{1}$.

Claim 1. There is a sequence of reals $\left\langle r_{\alpha}\right| \alpha\left\langle\lambda^{+}\right\rangle$in a generic ultrapower such that for every $s \subseteq \omega_{1}$ the final segment of $\left\langle r_{\alpha} \mid \alpha<\lambda^{+}\right\rangle$are Cohen (or random) generic over $L[s]$.

Proof: Let $N$ be a generic ultrapower. Then ${ }^{\kappa} N \subseteq N$ where ${ }^{\kappa} N$ is in the sense of the generic extension. First note that if $\left\langle s_{\alpha} \mid \alpha<\kappa\right\rangle$ is a sequence of $\kappa$ Cohen (random) reals over $V$, then it belongs to $N$. Clearly every $s \subseteq \omega_{1}$ in $N$ (or the same in $V[G]$ ) is a name in $V$ interpreted using $\aleph_{1}$ Cohen (random) reals only. Hence the final segment of $\left\langle s_{\alpha} \mid \alpha<\kappa\right\rangle$ will be generic over $L[s]$. Then, in $V$, for every regular $\delta<\kappa$ there will be a sequence $\left\langle t_{\alpha} \mid \alpha<\delta\right\rangle$ such that for every $s \subseteq \omega_{1}$ its final segment is Cohen (random) generic over $L[s]$. Back in $N$, we use this for $\delta=\lambda^{+}$which is still below $j(\kappa)$.

of the claim.

Let us fix such a sequence $\left\langle r_{\alpha} \mid \alpha<\lambda^{+}\right\rangle$in $N$. We split it into blocks each of the length $\omega_{1}$. Denote such changed sequence by $\left\langle r_{\alpha i} \mid \alpha<\lambda^{+}, i<\omega_{1}\right\rangle$. Now back in $V$, let us use the fact that $\operatorname{cov}\left(\lambda, \aleph_{2}, \aleph_{2}, \aleph_{1}\right)=\lambda$. We know that for every $\alpha<\lambda^{+}$the block $\left\langle\sim_{\alpha_{i}} \mid i<\omega_{1}\right\rangle$ is added by using only $\omega_{1}$ Cohen (or random) reals from the $\lambda$ Cohen (or Random) reals, $\left\langle C_{\beta} \mid \beta<\lambda\right\rangle$. More precisely, for every $\alpha<\lambda^{+}$there is $t_{\alpha}:{ }^{\omega} \omega_{1} \longrightarrow \omega_{>} \omega_{1}$ in $V$ and a set of indexes $b_{\alpha} \in V[G]$ and some enumeration of it $\left\langle\xi_{\alpha i} \mid i<\omega_{1}\right\rangle$ such that $\left\langle r_{\alpha i} \mid i<\omega_{1}\right\rangle$ is the image of $\left\langle C_{\xi_{\alpha_{i}}} \mid i<\omega_{1}\right\rangle$ under $t_{\alpha}$. Since $2^{<\kappa}<\lambda$, we can assume w.l. of $\mathrm{g}$. that for some $t$ for every $\alpha<\lambda^{+} t_{\alpha}=t$. We view $b_{\alpha}$ as $\left\langle\left\langle\xi_{\alpha i}, i\right\rangle \mid i<\omega_{1}\right\rangle$ i.e. a subset of $\lambda \times \omega_{1}$ of cardinality $\aleph_{1}$. Since $|\lambda|=\left|\lambda \times \omega_{1}\right|$ we still can use $\operatorname{cov}\left(\lambda, \omega_{2}, \omega_{2}, \omega_{1}\right)=\lambda$ and find $b \subseteq \lambda \times \omega_{1},|b|=\aleph_{1}$ such for $\lambda^{+} \alpha^{\prime} s\left|b \cap b_{\alpha}\right|=\aleph_{1}$ and $t$ applied to $b$ provides a nontrivial information about $\left\langle r_{\alpha i} \mid i<\omega_{1}\right\rangle$. But now the final segment of $\left\langle r_{\alpha i} \mid \alpha<\lambda^{+}, i<\omega_{1}\right\rangle$ cannot be Cohen (random) generic over $L[t, b]$. Contradiction.

\section{Another Construction of a Model with a Real-Valued Measurable Cardinal}

In this section we construct a model with a real-valued measurable cardinal which differs from the Solovay original. This answers negatively a question of D. Fremlin [Fr, (P1)]: 
Let $N$ be a model of ZFC and $\kappa \in N$ a real-valued measurable cardinal in $N$. Does it follow that there are inner models $M \subseteq N$ such that $\kappa$ is a measurable in $M$ and $M$-generic filter $G$ for a random real p.o. set over $M$ such that $G \in N$ and $N \cap \mathcal{P}(\kappa) \subseteq M[G]$.

Suppose that $\kappa$ is a measurable and $G C H$ holds. We define a forcing notion $P$ as follows:

Definition 2.1. $\quad P$ consists of all triples $p=\left\langle p_{0}, p_{1}, p_{2}\right\rangle$ so that

(1) $p_{0} \subseteq \kappa$

(2) $p_{1}$ is a function with domain contained in $p_{0}$

(3) $p_{2}$ is a function defined over inaccessibles $\leq \kappa$

(4) for every inaccessible $\delta\left|p_{0} \cap \delta\right|<\delta$, $\left|\operatorname{dom} p_{1} \cap \delta\right|<\delta$ and $\left|\operatorname{dom} p_{2} \cap \delta\right|<\delta$

(5) for every $\alpha \in \operatorname{dom} p_{1} p_{1}(\alpha) \subseteq \alpha$

(6) every element of $p_{0}$ is an ordinal of cofinality $\aleph_{0}$

(7) for every limit ordinal $\beta$ if $\operatorname{cf} \beta>\aleph_{0}$, then $p_{0} \cap \beta$ is not stationary in $\beta$ and if $\operatorname{cf} \beta=\aleph_{0}$ then $\beta \backslash(p \cap \beta)$ is unbounded in $\beta$

(8) for every $\alpha \in \operatorname{dom} p_{2} p_{2}(\alpha)$ is a closed subset of $\alpha$ disjoint with $p_{0}$.

Definition 2.2. $\quad$ Let $p, q \in P p=\left\langle p_{0}, p_{1}, p_{2}\right\rangle$ and $q=\left\langle q_{0}, q_{1}, q_{2}\right\rangle$. Then $p \geq q$ iff

(1) $p_{1} \subseteq q_{1}$

(2) $\operatorname{dom} p_{2} \supseteq \operatorname{dom} q_{2}$ and for every $\alpha \in \operatorname{dom} q_{2} p_{2}(\alpha)$ is an end extension of $q_{2}(\alpha)$

(3) $p_{0} \supseteq q_{0}$

(4) for every $\delta<\kappa, \delta$ is an inaccessible or a limit of inaccessibles and $\delta^{*}$ is the least inaccessible above $\delta$ then $p_{0} \cap\left[\delta, \delta^{*}\right)$ is an end extension of $q_{0} \cap\left[\delta, \delta^{*}\right)$.

The forcing $P$ is intended to add three objects. Thus, the first coordinates of $P$ are producing a subset $S$ of $\kappa$ which is stationary in $V[S]$ and reflecting only in inaccessibles. The second coordinate is responsible for a kind of diamond sequence over $S$ and the last coordinate adds clubs preventing reflection of $S$ at inaccessibles and its stationarity.

The forcing $P$ destroys the measurability of $\kappa$ once used over $V=L[\mu]$. It is bad for our purpose. We are going to use a certain subforcing of $P$ which will preserve measurability 
and contain the projection of $P$ to the first two coordinates. But first let us study basic properties of $P$.

Let $\left.P_{0}=\left\{p_{0} \mid \exists\left\langle p_{1}, p_{2}\right\rangle\left\langle p_{0}, p_{1}, p_{2}\right\rangle \in P\right\}, P_{01}=\left\langle\left\langle p_{0}, p_{1}\right\rangle\right| \exists p_{2}\left\langle p_{0}, p_{1}, p_{2}\right\rangle \in P\right\}$. Let $\alpha$ be an inaccessible. We denote by $P\lceil\alpha$ the set

$$
\left\{\left\langlep_{0} \cap \alpha, p_{1}\left\lceil\alpha, p_{2}\lceil\alpha\rangle \mid\left\langle p_{0}, p_{1}, p_{2}\right\rangle \in P\right\}\right.\right.
$$

and by $P \backslash \alpha$ the set

$$
\left\{\left\langlep_{0} \backslash \alpha, p_{2}\left\lceil[\alpha, \kappa), p_{2}\lceil[\alpha+1, \kappa)\rangle \mid\left\langle p_{0}, p_{1}, p_{2}\right\rangle \in P\right\}\right.\right.
$$

$P_{0}\left\lceil\alpha, P_{01}\left\lceil\alpha\right.\right.$ and $P_{0} \backslash \alpha, P_{01} \backslash \alpha$ are defined similarly.

The following is standard.

Claim 2.3. Let $\alpha$ be an inaccessible then the following holds

(1) $P=P\lceil\alpha \times P \backslash \alpha$

(2) $P_{0}=P_{0}\left\lceil\alpha \times P_{0} \backslash \alpha\right.$

(3) $P_{01}=P_{01}\left\lceil\alpha \times P_{01} \backslash \alpha\right.$.

Let $\alpha<\kappa$ be a limit ordinal and $Q$ a forcing notion.

Consider the following game $\operatorname{Game}(Q, \alpha)$ :

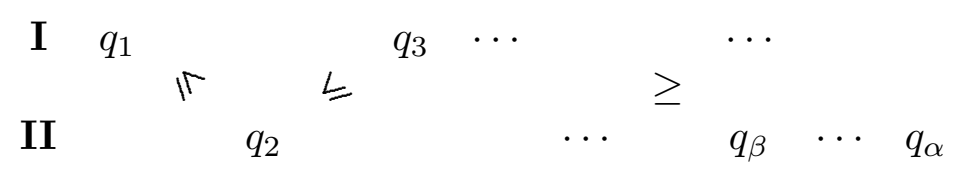

where Players I, II are building an increasing sequence of elements of $Q$, I at even stages and II at odds. If at some stage $\beta<\alpha$ II cannot continue i.e. there is no $q$ above $\left\{q_{\beta}^{\prime} \mid \beta^{\prime}<\beta\right\}$ then I wins. Otherwise II wins.

Claim 2.4. The player II has a winning strategy in the game Game $\left(P \backslash \alpha, \alpha^{+}\right)$for every inaccessible $\alpha$.

Proof: Let $\alpha$ be an inaccessible. We define a winning strategy $\sigma$ for Player II in the Game $\left(P \backslash \alpha, \alpha^{+}\right)$. 
Let $\delta>\alpha$ be an inaccessible but not limit one. Denote by $\delta^{-}$the supremum of inaccessibles below $\delta$.

Let $p \in P \backslash \alpha$. We define $\bar{p}$ to be the condition obtained from $p=\left\langle p_{0}, p_{1}, p_{2}\right\rangle$ by adding $\sup \left(p_{0} \cap\left[\delta^{-}, \delta\right)\right)+\sup \left(p_{2}(\delta)\right)$ to $p_{2}(\delta)$ if $p_{0} \cap\left[\delta^{-}, \delta\right) \neq \emptyset$ or $p_{0} \cap\left[\delta^{-}, \delta\right)=\emptyset$ but $p_{0} \cap \delta^{-}$is unbounded in $\delta^{-}$, where $\delta$ runs over inaccessibles above $\alpha$ which are not limit inaccessibles and $\sup \left(p_{2}(\delta)\right)=0$ whenever $\delta \notin \operatorname{dom} p_{2}$.

Now we define $\sigma$ to be dependent only on the last move of $I$ at successive stages of the game. Set $\sigma\left(p_{\beta+1}\right)=\bar{p}_{\beta+1}$. If $\beta \leq \alpha^{+}$is limit and the game up to $\beta$

$$
\begin{array}{lllll}
p_{1} & & p_{3} & \cdots & \\
& p_{2} & & \cdots & p_{\gamma}
\end{array}
$$

was played according to $\sigma$. Then set $\sigma\left(\left\langle p_{\gamma} \mid \gamma<\beta\right\rangle\right)=$ the closure of $\bigcup_{\gamma<\beta} p_{\gamma}$. More precisely, let $\sigma\left(\left\langle p_{\gamma} \mid \gamma<\beta\right\rangle\right)=\left\langle p^{0}, p^{1}, p^{2}\right\rangle$ where $p^{0}=\bigcup_{\gamma<\beta} p_{\gamma}^{0}, p^{1}=\bigcup_{\gamma<\beta} p_{\gamma}^{1}$ and $\operatorname{dom}\left(p^{2}\right)=\bigcup_{\gamma<\beta} \operatorname{dom}\left(p_{\gamma}^{2}\right), p^{2}(\xi)=\bigcup\left\{p_{\gamma}^{2}(\xi) \mid \gamma<\beta, \xi \in \operatorname{dom} p_{\gamma}^{2}\right\} \cup\left\{\sup \left(\cup\left\{p_{\gamma}^{2}(\xi) \mid \xi \in\right.\right.\right.$ $\left.\left.\left.\operatorname{dom} p_{2}^{\gamma}, \gamma<\beta\right\}\right)\right\}$,for $\xi \in \operatorname{dom} p^{2}$.

We need to check that such defined $p$ is a condition. The only problem is to show that $p^{0}$ does not reflect at any $\tau, \aleph_{0}<c f \tau<\tau$. So let $\tau$ be an ordinal such that $\aleph_{0}<c f \tau<\tau$ and $p^{0} \cap \tau$ is unbounded in $\tau$. Pick $\delta$ to be the first inaccessible above $\tau$. Then $\delta^{-} \leq \tau$. If $\delta^{-}<\tau$, then starting with some $\gamma_{0}<\beta \quad p_{\gamma}^{0} \cap\left[\delta^{-}, \delta\right) \neq \emptyset$. But then $p^{2}(\delta)$ will be a club of $\tau$ disjoint to $p^{0} \cap \tau$. Suppose now that $\delta^{-}=\tau$. Then $C_{0}=\left\{\sup \left(\bigcup_{\gamma^{\prime}<\gamma}\left(p_{\gamma^{\prime}}^{0} \cap \tau\right)\right) \mid\right.$ $\gamma<\beta, \gamma$ limit $\}$ is a club of $\tau$. Also $C_{1}=\{\xi<\tau \mid \xi$ is a limit of inaccessibles $\}$ is a club of $\tau$. Let $\xi \in C_{0} \cap C_{1}$. Then for some inaccessible $\underline{\delta}<\tau \xi=\underline{\delta}^{-}$. Let $\gamma_{\xi}<\beta$ be so that $\xi=\sup \bigcup_{\gamma^{\prime}<\gamma_{\xi}}\left(p_{\gamma^{\prime}}^{0} \cap \tau\right)$. Then, $\xi \in p_{\gamma_{\xi}}^{2}(\underline{\delta})$, by the definition of $\sigma$ and the procedure. Thus, this provides a club of $\tau$ disjoint to $p_{\beta}^{0} \cap \tau$. $\square$ of the claim.

The following is now trivial.

Claim 2.5. $P$ preserves cofinalities and does not add new functions from ordinals less than the first inaccessible into $V$.

Let $U$ be a normal measure over $\kappa$ and $j: V \rightarrow N$ the corresponding elementary embedding. Then, in $N, j(P)=j(P) \mid \kappa \times j(P) \backslash \kappa$. Clearly, $\left(j(P)\lceil\kappa)^{N}=P\right.$. Now let us produce inside $V$ an $N$-generic subset of $j(P) \backslash \kappa$ with the set over the first coordinate nonstationary in $V$. 
Claim 2.6. There exists $\left\langle S, F_{1}, F_{2}\right\rangle$ such that

(a) $\left\langle S, F_{1}, F_{2}\right\rangle$ is $j(P) \backslash \kappa^{+}$generic over $N$

(b) $S$ is not stationary subset of $j(\kappa)$

(c) $S$ does not reflect.

Proof: Let $\left\langle D_{\alpha} \mid \alpha<\kappa^{+}\right\rangle$be the list of dense open subsets of $j(P) \backslash \kappa$ of $N$. Let $\sigma \in N$ be a winning strategy for Player II in Game $\left(j(P) \backslash \kappa, \kappa^{+}\right)$. It exists by Claim 2.4 applied in $N$ to $j(P)$. Play the game from $V$ so that I plays at stage $\beta+1$ an element $P_{\beta+1}$ of $D_{\beta}$ which is above $p_{\beta}$, where $\beta<\kappa^{+}$. We will finish with a desired $N$-generic set.

Force with $P$ over $V$. Let $G$ be a generic subset. We denote $\bigcup\left\{p_{0} \mid \exists\left\langle p_{1}, p_{2}\right\rangle\left\langle p_{0}, p_{1}, p_{2}\right\rangle\right.$ $\in G\}$ by $S$. For every $\alpha \in S$ let $A_{\alpha}=\bigcup\left\{p_{1}(\alpha) \mid \exists\left\langle p_{0}, p_{1}, p_{2}\right\rangle \in G\right.$ and $\left.\alpha \in \operatorname{dom} p_{1}\right\}$ and for inaccessible $\delta \leq \kappa$ let $C_{\delta}=\bigcup\left\{p_{2}(\delta) \mid \exists\left\langle p_{0}, p_{1}, p_{2}\right\rangle \in G\right.$ and $\left.\delta \in \operatorname{dom} p_{2}\right\}$. Then $S \subseteq \kappa$, and for every inaccessible $\delta \leq \kappa C_{\delta}$ is a club of $\delta$ disjoint to $S$.

Claim 2.7. $S$ is a stationary nonreflecting subset of $\kappa$ in $V\left[S,\left\langle A_{\alpha} \mid \alpha \in S\right\rangle,\left\langle C_{\delta}\right|\right.$ $\delta$ inaccessible and $\delta<\kappa\rangle]$.

Proof: $\left\langle C_{\delta} \mid \delta<\kappa\right\rangle$ are witnessing the nonreflection. Suppose that $S$ is nonstationary in $V\left[S,\left\langle A_{\alpha} \mid \alpha \in S\right\rangle,\left\langle C_{\delta}\right| \delta\right.$ inaccessible and $\left.\left.\delta<\kappa\right\rangle\right]$. Return back to $V$ and work with names. Suppose for simplicity that the empty condition forces the nonstationarity of $S$. Let $\underset{\sim}{C}$ be a name of witnessing club. Pick an elementary submodel $N$ of $V_{2^{2^{\kappa}}}$ such

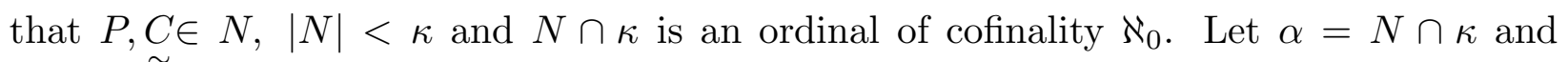
$\left\langle\alpha_{n} \mid n<\omega\right\rangle$ be a cofinal in $\alpha$ sequence. Now by induction we construct an increasing sequence $\left\langle p_{i} \mid i<\omega\right\rangle$ of conditions of $P\lceil\kappa$ (i.e. $P$ without the information on a club of $\kappa$ disjoint to $S$ ) such that for every $i<\omega$

(a) $p_{i} \in N$

(b) $p_{i}$ decides the first element of $\underset{\sim}{C}$ above $\alpha_{i}$

(c) $\sup \left(p_{i}\right)_{0} \geq \alpha_{i}$.

where $p_{i}=\left\langle\left(p_{i}\right)_{0},\left(p_{i}\right)_{1},\left(p_{i}\right)_{2}\right\rangle$ 
Now, in $V$, let

$$
p=\left\langle\bigcup_{i<\omega}\left(p_{i}\right)_{0} \cup\{\alpha\}, \bigcup_{i<\omega}\left(p_{i}\right)_{1},\left\{\left\langle\delta, \cup\left\{\left(p_{i}\right)_{2}(\delta) \mid i<\omega, \delta \in \operatorname{dom}\left(p_{i}\right)_{2}\right\}\right\rangle .\right.\right.
$$

Then $p \Vdash \alpha \in \underset{\sim}{\cap} \cap \underset{\sim}{S}$. Contradiction.

Claim 2.8. $\quad \kappa$ is a measurable cardinal in $V\left[S,\left\langle A_{\alpha} \mid \alpha \in S\right\rangle\right]$.

Proof: Just note that in $N j\left(P_{01}\right)=P_{01} \times j\left(P_{01}\right) \backslash \kappa^{+}$, since nothing is done in the interval $\left[\kappa, \kappa^{+}\right]$by this forcing. By Claim 2.6, there is a $j\left(P_{01}\right) \backslash \kappa^{+}$generic over $N$ set in $V$. Thus it is easy to extend $j$ to the embedding of $V\left[S,\left\langle A_{\alpha} \mid \alpha \in S\right\rangle\right]$. This insures the measurability of $\kappa$.

Notice, that $\kappa$ is not measurable in $V\left[S,\left\langle A_{\alpha} \mid \alpha \in S\right\rangle,\left\langle C_{\delta}\right| \delta<\kappa, \delta\right.$ inaccessible $\left.\rangle\right]$ since $S$ is a stationary nonreflecting subset of $\kappa$.

Now, over a model $V\left[S,\left\langle A_{\alpha} \mid \alpha \in S\right\rangle\right]$ we are going to force a Boolean algebra $B$ such that:

(a) $\kappa$ is still measurable in $V\left[S,\left\langle A_{\alpha} \mid \in S\right\rangle, B\right]$

(b) $j^{*}(B) / G(B)$ is isomorphic to the adding of $j(\kappa)$-Random reals, where $j^{*}$ the elementary embedding of $V\left[S,\left\langle A_{\alpha} \mid \alpha \in S\right\rangle, B\right]$ into its ultrapower and $G(B)$ a generic subset of $B$.

First let us review some basics of product measure algebras. We refer to D. Fremlin [Fr2] for detailed presentation.

Suppose that $B$ is a $\sigma$-algebra, i.e. a Boolean algebra all of whose countable suprema exist. A measure on $B$ is a function $\mu: B \rightarrow[0,1]$ so that: (a) $\mu\left(1_{B}\right)=1$, and (b) whenever $\left\{b_{n} \mid n \in \omega\right\} \subseteq B$ with $b_{n} \wedge b_{n}=0$ for $n \neq m$, then $\mu\left(V_{n} b_{n}\right)=\sum_{n} \mu\left(b_{n}\right)$. If in addition $\mu$ is positive (i.e. $\mu(b)=0$ iff $b=0$ ), then we say that $\langle B, \mu\rangle$ is a measure algebra. A measure algebra is always a complete Boolean algebra.

Suppose now that $I$ is a set, and $\left\langle B_{i}, \mu_{i}\right\rangle$ for $i \in I$ are measure algebras. Call $C \in \prod_{i \varepsilon I} B_{i}$ a cylinder iff $C(i)$ is the unit element of $B_{i}$, except for a finite number of coordinates $i$. Let $B \supseteq \prod_{i \varepsilon I} B_{i}$ be the $\sigma$-algebra generated by the cylinders. It is known that there is a 
unique measure $\mu$ on $B$ so that $\mu(C)=\prod_{i \varepsilon I} \mu_{i}(C(i))$ for any cylinder $C$. $\mu$ may not be positive, but there is a standard strategy: Let $I=\{b \in B \mid \mu(b)=0\}$. Then $I$ is an ideal, and $\bar{B}=B / I$ as usual is a $\sigma$-algebra consisting of equivalence classes $[b]$ for $b \in B$ (where $[b]=[c]$ iff the symmetric difference $(b-c) \vee(c-b) \varepsilon I)$. We can define a positive measure $\bar{\mu}$ on $\bar{B}$ by: $\bar{\mu}([b])=\mu(b)$. Thus, $\langle\bar{B}, \bar{\mu}\rangle$ is a measure algebra, called the product measure algebra of the $\left\langle B_{i}, \mu_{i}\right\rangle$ 's.

Let 2 be the basic measure algebra $\langle P(2), \mu\rangle$ where $\mu$ is the measure: $\mu(\emptyset)=0, \mu(\{0\})=$ $\mu(\{1\})=\frac{1}{2}$, and $\mu(\{0,1\})=1$. For any set $I$, let $\mathbf{2}^{I}$ denote the product measure algebra of $I$ copies of $\mathbf{2}$. We can then force with $\mathbf{2}^{I}$ with the natural proviso: $b$ is a stronger condition than $c$ iff $0<b \leq c$ in $\mathbf{2}^{I}$. This forcing obviously has the $\omega_{1}$-c.c.

For $I=\omega, \mathbf{2}^{I}$ is just the usual random real forcing and for $I=\lambda \mathbf{2}^{I}$ is the $\lambda$-random real forcing. Let us denote them by Random and Random $(\lambda)$ respectively.

We consider the $\sigma$-algebras $B_{\alpha} \subseteq \prod_{i<\alpha}(\mathcal{P}(2))_{i}$ generated by the cylinders, where $\alpha \leq \kappa$ and $(\mathcal{P}(2))_{i}$ is just $i$-th copy of $\mathcal{P}(2)$. The desired algebra $B$ will be $B_{\kappa} / I_{\kappa}$, where the ideal $I_{\kappa}$ of "null" sets is going to be added generically. More precisely for $\alpha$ 's of countable cofinality $I_{\alpha}$ 's will be added by forcing and then for $\beta \leq \kappa$ of uncountable cofinality $I_{\beta}$ will the union of $I_{\alpha}$ 's, where $\alpha<\beta, c f \alpha=\aleph_{0}$. The sequence of ideals $\left\langle I_{\alpha} \mid \alpha \leq \kappa\right\rangle$ will be in $V_{1}=V\left[S,\left\langle A_{\alpha} \mid \alpha \in S\right\rangle,\left\langle C_{\delta}\right| \delta<\kappa, \delta\right.$ inaccessible $\left.\rangle\right]$.

Let us work in $V_{1}$. We define by induction on $\alpha<\kappa$ a measure $\mu_{\alpha}$ on $B_{\alpha}$. Then $I_{\alpha}$ will be the ideal of $\mu_{\alpha}$-measure null sets. Actually there will be a lot of different measures over $B_{\alpha}$ 's. We would like to prevent $B_{\kappa}$ (and even its subalgebras of power $\kappa$ ) from carrying a measure. For this purpose, the "diamond" sequence $\left\langle A_{\alpha} \mid \alpha \in S\right\rangle$ will be used to destroy possible candidates.

If $\alpha<\min S$, then let $\mu_{\alpha}$ be the usual product measure over $B_{\alpha}$, i.e. one generated by attaching weight $1 / 2$ to $\{0\}$ and $\{1\}, 0$ to $\emptyset$ and 1 to $\{0,1\}$ in every component $(\mathcal{P}(2))_{i}$ $(i<\alpha)$ of the product $\prod_{i<\alpha}(\mathcal{P}(2))_{i}$. Set $I_{\alpha}=\left\{X \in B_{\alpha} \mid \mu_{\alpha}(X)=0\right\}$.

Suppose now that $\alpha<\kappa$ and for every $\beta<\alpha$ the measure $\mu_{\beta}$ over $B_{\beta}$ was already defined. We need to define $\mu_{\alpha}$ over $B_{\alpha}$.

Case 1. $\alpha \notin S$.

Pick an increasing continuous sequence $\left\langle\alpha_{\tau} \mid \tau<c f \alpha\right\rangle$ witnessing nonstationarity of $S \cap \alpha$. 
In case, $c f \alpha=\aleph_{0}$ just use $\omega$-sequence unbounded in $\alpha$ and disjoint with $S$. For every $\tau<c f \alpha$ let $\mu(\tau)$ be $\mu_{\alpha_{\tau+1}} \uparrow\left(B_{\alpha_{\tau+1}}\left\lceil\left[\alpha_{\tau}, \alpha_{\tau+1}\right)\right)\right.$, where $B_{\alpha_{\tau+1}} \uparrow\left[\alpha_{\tau}, \alpha_{\tau+1}\right)$ is the subalgebra of $\left.\prod_{\alpha_{\tau} \leq i<\alpha_{\tau+1}}(\mathcal{P}(2))\right)_{i}$ generated by the cylinders.

Let $\mu_{\alpha}$ be the product measure of $\left\langle<B_{\alpha_{\tau+1}} \uparrow\left[\alpha_{\tau}, \alpha_{\tau+1}\right), \mu(\tau)>\mid \tau<c f \alpha\right\rangle$.

Notice that $\alpha_{\tau} \notin S$. Therefore by induction, we can assume that for a limit $\tau$ the measure $\mu_{\alpha_{\tau}}$ over $B_{\alpha_{\tau}}$ is the product measure of $\left\langle<B_{\alpha_{\nu}} \uparrow\left[\alpha_{\nu}, \alpha_{\nu+1}\right), \mu(\nu)\right\rangle|\nu<\tau\rangle$.

Case 2. $\alpha \in S$.

Suppose that $A_{\alpha}$ codes in some reasonable fashion sequences $\left\langle\alpha_{n} \mid<\omega\right\rangle,\left\langle\varphi_{n} \mid n<\omega\right\rangle$, $\langle\mu(n) \mid n<\omega\rangle$ and $\left\langle a_{n} \mid n<\omega\right\rangle$ so that for every $n<\omega$

(a) $\left\langle\alpha_{n} \mid n<\omega\right\rangle$ is a cofinal in $\alpha$ sequence

(b) $a_{n}$ is a countable subset of $\left[\alpha_{n}, \alpha_{n+1}\right)$

(c) $\mu(n)$ is a measure over $B_{\alpha_{n+1}}\left\lceil a_{n}\right.$ respecting the ideal $I_{\alpha_{n+1}}\left\lceil a_{n}\right.$, i.e. for every $X \in$ $B_{\alpha_{n+1}}\left\lceil a_{n} \mu(n)(X)=0\right.$ iff $X \in I_{\alpha_{n+1}}$

(d) $\varphi_{n}$ : Random $\leftrightarrow B_{\alpha_{n+1}}\left\lceil a_{n}\right.$ is a measure algebra isomorphism.

Denote $B_{\alpha_{n+1}}\left\lceil a_{n}\right.$ by $B(n)$. Let us define a measure $\widetilde{\mu}(n)$ over $B(n)$. Thus for every $n<\omega$ let us change the value $\mu(n)\left(\varphi_{n}(\{0\})\right)$ from $1 / 2$ to $1-\frac{1}{\pi^{2} n^{2}}$ and those of $\varphi_{n}(\{1\})$ from $1 / 2$ to $\frac{1}{\pi^{2} n^{2}}$. Let $\widetilde{\mu}(n)$ be the measure obtained from $\mu(n)$ in such a fashion. Clearly, such local changes have no effect on the set of measure zero. Namely, for every $X \in B(n)$ $\mu(n)(X)=0$ iff $\widetilde{\mu}(n)(X)=0$.

Define now the measure $\mu_{\alpha}$ over $B_{\alpha}$ as the product measure of the measure algebras $\langle B(n), \widetilde{\mu}(n)\rangle(n<\omega)$ together with all the rest, i.e.

$$
\left\langle B_{\alpha_{n+1}} \uparrow\left(\left[\alpha_{n}, \alpha_{n+1}\right) \backslash a_{n}\right), \mu_{\alpha_{n+1}} \uparrow\left(\left[\alpha_{n}, \alpha_{n+1}\right) \backslash a_{n}\right)\right\rangle .
$$

We claim that $\varphi=\bigcup_{n<\omega} \varphi_{n}$ cannot be extended to complete embedding into $\left\langle B_{\alpha}, \mu_{\alpha}\right\rangle$. The reason is that under $\varphi$ the measure of the set $\bigcap_{n<\omega} \varphi_{n}(\{0\})$ should be zero, but $\mu_{\alpha}\left(\bigcap_{n<\omega} \varphi_{n}(\{0\})\right)=\prod_{n<\omega} \widetilde{\mu}(n)\left(\varphi_{n}(\{0\})\right)=\prod_{n<\omega}\left(1-\frac{1}{\pi^{2} n^{2}}\right)$ which equals $\sin (1) \neq 0$ by the Euler formula.

Notice, that the ideal $I_{\alpha}$ of $\mu_{\alpha}$-measure zero sets will not be effected if for finitely many $n$ 's the measures $\mu(n)$ will be used in the product instead of $\widetilde{\mu}(n)$ 's. Also, if in the previous 
construction we will do everything above some $\alpha_{n_{o}}$ for fixed $n_{0}<\omega$, i.e. we will define the measure over $B_{\alpha}\left\lceil\left[\alpha_{n_{0}}, \alpha\right)\right.$ instead of all $B_{\alpha}$ call it $\mu_{\alpha}\left\lceil\left[\alpha_{n_{0}}, \alpha\right)\right.$ and its ideal $I_{\alpha}\left\lceil\left[\alpha_{n_{0}}, \alpha\right)\right.$, then, for every $X \in B_{\alpha} X \in I_{\alpha}$ iff $X\left\lceil\alpha_{n_{0}} \in I_{\alpha_{n_{0}}}\right.$ and $X\left\lceil\left[\alpha_{n_{0}}, \alpha\right) \in I_{\alpha}\left\lceil\left[\alpha_{n_{0}}, \alpha\right)\right.\right.$. This means that once having $I_{\alpha_{n}}$ 's, initial segments of measures $\langle\widetilde{\mu}(n) \mid n<\omega\rangle$ have no effect on $I_{\alpha}$. This observation will be crucial further for showing measurability of $\kappa$.

If $A_{\alpha}$ does not guess the sequences as above, then we proceed as in Case 1.

This completes the definition of $\left\langle\mu_{\alpha} \mid \alpha<\kappa\right\rangle$ and hence also $\left\langle I_{\alpha} \mid \alpha \leq \kappa\right\rangle$.

We set $B=B_{\kappa} / I_{\kappa}$. Let $V_{2}=V\left[S,\left\langle A_{\alpha} \mid \alpha \in S\right\rangle,\left\langle I_{\alpha} \mid \alpha<\kappa, \operatorname{cf} \alpha=\aleph_{0}\right\rangle\right]$. Then for every $\alpha<\kappa I_{\alpha} \in V_{2}$. So $B \in V_{2}$. We will show the following claim which has a proof similar to 2.7 .

Claim 2.9. Random $(\kappa)$ does not embed into $B$ in $V_{1}$ and also in $V_{2}$.

Proof: Notice that $V_{2}$ and $V_{1}$ have the same reals. So if $\varphi$ is an embedding of $\operatorname{Random}(\kappa)$ into $B$ in $V_{2}$ then $\varphi$ will be also such embedding in $V_{1}$. Hence let us prove the claim for $V_{1}$.

Suppose otherwise. Let $\varphi: \operatorname{Random}(\kappa) \longrightarrow B$ witnessing embedding. Back in $V$ let us work with names. Let $\underset{\sim}{\varphi}$ be a name of $\varphi$ and assume for simplicity that the empty condition forces this.

Pick $N$ and $\left\langle\alpha_{n} \mid n<\omega\right\rangle$ to be as in Claim 2.7 with $\varphi$ replacing $\widetilde{C}$.

We define sequences of conditions of $P\left\lceil\kappa\right.$ of $\left\{p_{n} \mid n<\omega\right\} \subseteq N$, of ordinals $\left\langle\beta_{n}\right| n<$ $\omega\rangle$, countable sets $\left\langle a_{n} \mid n<\omega\right\rangle$ and embedding $\left\langle\varphi_{n} \mid n<\omega\right\rangle$ so that

(a) $\sup \left(p_{n}\right)_{0} \geq \alpha_{n}$

(b) $\beta_{n} \geq \alpha_{n}$

(c) $p_{n} \Vdash \longmapsto^{\prime \prime} \underset{\sim}{\varphi}\left(\{0\} \beta_{n}\right)$ has nontrivial intersection with $\underset{\sim}{B} \uparrow\left[\beta_{n}, \beta_{n+1}\right)^{\prime \prime}$, where $\{0\}_{\beta_{n}} \in$ $(\mathcal{P}(2))_{\beta_{n}}$ i.e. the $\beta_{n}$-th copy of $\mathcal{P}(2)$.

(d) $a_{n} \subseteq \beta_{n}$

(e) $\varphi_{n}$ embeds $(\mathcal{P}(2))_{\beta_{n}}$ into $B_{\kappa}\left\lceil a_{n}\right.$

(f) $p_{n} \Vdash \longleftarrow^{\prime \prime} \check{\varphi}_{n}$ is equal to $\underset{\sim}{\varphi} \uparrow(\mathcal{P}(2))_{\beta_{n}} \bmod {\underset{\sim}{\kappa}}_{\kappa}{ }^{\prime \prime}$

Since the forcing does not add new countable sequences of elements of $V$, there is no problem in carrying out the induction. 
Denote by $\mu(n)$ the measure over $B_{\kappa}\left\lceil a_{n}\right.$ induced by $\varphi_{n}$.

Now let $A_{\alpha} \subseteq \alpha$ be a code for such sequences

$$
\left\langle\beta_{n} \mid n<\omega\right\rangle,\left\langle a_{n} \mid n<\omega\right\rangle,\left\langle\varphi_{n} \mid n<\omega\right\rangle
$$

and $\langle\mu(n) \mid n<\omega\rangle$.

Set $p=\left\langle\bigcup_{n<\omega}\left(p_{n}\right)_{0} \cup\{\alpha\}, \bigcup_{n<\omega}\left(p_{n}\right)_{1} \cup\left\{\left\langle\alpha, A_{\alpha}\right\rangle\right\}\right.$,

$$
\left\{\left\langle\delta, \cup\left\{\left(p_{n}\right)_{2}(\delta) \mid n<\omega, \delta \in \operatorname{dom}\left(p_{n}\right)_{2}\right\}\right\rangle\right.
$$

Then $p \Vdash{ }^{\prime \prime} \varphi$ does not embed $\prod_{n<\omega}(\mathcal{P}(2))_{\beta_{n}}$ into $B_{\alpha} / I_{\sim} \alpha^{\prime \prime}$, by the definition of $I_{\alpha}^{\prime \prime}$. Hence, also $p \Vdash \longmapsto^{\prime \prime} \varphi$ does not embed Random $(\kappa)$ into $B^{\prime \prime}$. Contradiction.

Claim 2.10. $\quad \kappa$ is a measurable cardinal in $V_{1}$.

Proof: Let $j: V \longrightarrow N$ be an elementary embedding witnessing the measurability of $\kappa$. We like to extend it to an embedding

$$
\begin{gathered}
\left.j^{*}: V\left[S,\left\langle A_{\alpha} \mid \alpha \in S\right\rangle,\left\langle I_{\alpha}\right| \alpha<\kappa \text { and } \operatorname{cf} \alpha=\aleph_{0}\right\rangle\right] \\
\left.\longrightarrow N\left[S^{*},\left\langle A_{\alpha} \mid \alpha \in S^{*}\right\rangle,\left\langle I_{\alpha}\right| \alpha<j(\kappa) \text { and } \operatorname{cf} \alpha=\aleph_{0}\right\rangle\right] .
\end{gathered}
$$

By Claim 2.6, $j$ extends to

$$
j^{\prime}: V\left[S,\left\langle A_{\alpha} \mid \alpha \in S\right\rangle\right] \longrightarrow N\left[S^{*},\left\langle A_{\alpha} \mid \alpha \in S^{*}\right\rangle\right]
$$

where $\left\langle S^{*} \backslash S,\left\langle A_{\alpha} \mid \alpha \in S^{*} \backslash S>\right\rangle \in V\right.$ is $j\left(P_{01}\right) \backslash \kappa$ generic over $N$. We like to produce ideals $\left\langle I_{\alpha}\left\lceil\left(B_{j(\kappa)}\left\lceil\lceil\kappa, j(\kappa))\left|\kappa<\alpha<j(\kappa), \operatorname{cf} \alpha=\aleph_{0}\right\rangle\right.\right.\right.\right.$ generic over $N$ but in $V$. In order to define $\left\langle I_{\alpha}\right| \alpha<\kappa$, cf $\left.\alpha=\aleph_{0}\right\rangle$ we used clubs witnessing nonreflection of $S$, i.e. $\left\langle C_{\delta}\right| \delta<\kappa, \delta$ inaccessible $\rangle$. By Claim 2.6, the only club which is needed in order to extend $j$ but is missing in $V$ is $C_{\kappa}$. But, we define generically $I_{\alpha}$ 's only for $\alpha$ 's of cofinality $\aleph_{0}$ and moreover initial segments have no influence on such $I_{\alpha}$ 's. This means that the definition of $\left\langle I_{\alpha}\left\lceil B_{j(\kappa)}\left\lceil[\kappa, j(\kappa))\left|\kappa<\alpha<j(\kappa), c f \alpha=\aleph_{0}\right\rangle\right.\right.\right.$ can be carried out completely inside $N\left[S^{*} \backslash S,\left\langle A_{\alpha} \mid \alpha \in S^{*} \backslash S\right\rangle,\left\langle C_{\delta}\right| \kappa<\delta<j(\kappa), \delta\right.$ inaccessible $\left.\rangle\right]$. All the sets $S^{*} \backslash S,\left\langle A_{\alpha} \mid \alpha \in S^{*} \backslash S\right\rangle$ and $\left\langle C_{\delta}\right| \kappa<\delta<j(\kappa), \delta$ inaccessible of $\left.N\right\rangle$ can be found inside 
$V$ by Claim 2.6. Hence we have enough sets to extend $j$ to $j^{*}$. Thus, the measurability of $\kappa$ is preserved in $V_{2}=V\left[S,\left\langle A_{\alpha} \mid \alpha \in S\right\rangle,\left\langle I_{\alpha}\right| \alpha<\kappa\right.$ and $\left.\left.\operatorname{cf} \alpha=\aleph_{0}\right\rangle\right]$.

Let $j^{*}: V_{2} \longrightarrow N_{2}=N\left[S^{*},\left\langle A_{\alpha} \mid \alpha \in S^{*}\right\rangle,\langle I \alpha| \alpha<j(\kappa)\right.$ and $\left.\left.\operatorname{cf} \alpha=\aleph_{0}\right\rangle\right]$ be the embedding of Claim 2.10.

Claim 2.11. $j^{*}(B) \uparrow[\kappa, j(\kappa))$ is isomorphic to $\operatorname{Random}\left(\kappa^{+}\right)$in $V_{2}$.

Proof: By Claim 2.6, there are in $V$ and hence in $V_{2}$ clubs $\left\langle C_{\delta}\right| \kappa<\delta \leq j(\kappa), \delta$ is an inaccessible in $N\rangle$ witnessing nonreflection of $S^{*} \backslash S$ in every $N$-inaccessible $\delta \leq j(\kappa)$. Using them we define measures $\mu_{\alpha}$ over $B_{\alpha}\left\lceil[\kappa, \alpha)\right.$ agreeing with ideals $I_{\alpha}$ for every $\alpha, \kappa<\alpha \leq$ $j(\kappa)$ as it was done for $B_{\alpha}$ 's below $\kappa$ in $V_{1}$. The final measure $\mu_{j(\kappa)}$ will turn $B_{j(\kappa)}\lceil\lceil\kappa, j(\kappa))$ into measure algebra. Since $|j(\kappa)|=\kappa^{+}$, by Maharam theorem, see [Fr2] we obtain the desired result.

So we have the following:

Theorem 2. $\kappa$ be the measurable cardinal of $L[\mu]=V$ (the minimal model with a measurable). Then $\kappa$ is a real valued measurable in $V_{2}^{B}$ but for every submodel $V^{\prime}$ of $V_{2}^{B}$ if $\kappa$ is a measurable in $V^{\prime}$, then there is no $G \in V_{2}^{B}$ which is Random $(\kappa)$ generic over $V^{\prime}$.

Proof: Suppose that $V^{\prime} \subseteq V_{2}^{B}, \kappa$ is a measurable in $V^{\prime}, G \subseteq \operatorname{Random}(\kappa)$ generic over $V^{\prime}$ and $G \in V_{2}^{B}$. But then $G$ is also $\operatorname{Random}(\kappa)$ generic over $V=L[\mu]$, since $L[\mu] \subseteq V^{\prime}$ by its minimality. But $V$ and $V_{2}$ have the some countable sequences of ordinals. So, $G$ will be $\operatorname{Random}(\kappa)$-generic also over $V_{2}$. This means that Random $(\kappa)$ embeds $B$, which is impossible by Claim 2.9 .

\section{The Forcing with Ideal Cannot be Isomorphic to Cohen $\times$ Random or Random $\times$ Cohen}

The result for $\kappa$-complete ideals over $\kappa$ was proved in [Gi-Sh1]. Max Bruke pointed out that the application of this in [Gi-Sh2] requires the result also for less than $\kappa$ complete ideals as well. The purpose of this section is to close this gap.

Theorem 3.1. Suppose that $I$ is a $\omega_{1}$-complete ideal over some $\kappa$ then the forcing with ideal (i.e. $\mathcal{P}(\kappa) / I$ ) cannot be isomorphic to Cohen $\times$ Random or Random $\times$ Cohen.

Proof: Let us deal with Random $\times$ Cohen case. The Cohen $\times$ Random case is similar. 
Suppose otherwise. $\mathcal{P}(\kappa) / I \simeq$ Random $\times$ Cohen. Without loss of generality for some $\kappa_{1} \leq \kappa$ and $f: \kappa \rightarrow \kappa_{1} \kappa \longmapsto_{\mathcal{P}(\kappa) / I} " \kappa_{1}$ is the critical point of the generic embedding and $\tilde{f}$ represents $\kappa_{1}$ in the ultrapower". Define an ideal $J$ over $\kappa_{1}$ to be the set of all $A \subseteq \kappa_{1}$ such that $f^{-1 \prime \prime}(A) \in I$. Denote $Q=\mathcal{P}(\kappa) / I$ and $Q_{1}=\mathcal{P}\left(\kappa_{1}\right) / J$. Then $Q_{1}$ is a complete subordering of $Q$. We define a $Q_{1}$-name $\underset{\sim}{\tau=}=\left\{\eta \in \omega>2 \mid\right.$ the condition $\left(\mathbb{1}_{\text {Random }}, \eta\right)$ is compatible with every element of $\left.\underset{\sim}{G}\left(Q_{1}\right)\right\}$. For $\eta, \nu \in \omega>2$ let us write $\eta \triangleright \nu$ if the sequence $\eta$ extends the sequence $\nu$. The following two claims are obvious.

Claim 3.2. $\quad \underset{\sim}{\sim}$ is a $Q_{1}$-name of a nonempty subset of $\omega>2$ closed under initial segments with no $\triangleleft$-maximal element and hence a tree.

Claim 3.3. $\Downarrow_{Q}^{\prime \prime}$ the Cohen real is an $\omega$-branch of $\underset{\sim}{ }$ ".

Claim 3.4. There is no $p \in Q_{1}$ and $\eta \in{ }^{\omega>} 2$ such that $p \longmapsto_{Q_{1}}$ "for every $v \in{ }^{\omega>} 2 v \triangleright \eta$ implies $v \in \tau_{\sim}^{\prime \prime}$.

Proof: Suppose otherwise. Let $p, \eta$ be witnessing this. Then above $p$ the forcing notion $Q_{1}$ is a complete subordering of Random. But it has to add a real. Hence it is isomorphic to Random which is impossible by [Gi-Sh1].

Let $T^{*}=\left\{T \mid T \subseteq \omega>2\right.$ is a tree and for every $n<\omega, \eta \in{ }^{n} 2$ there are $v \triangleright \eta$ and $k<\omega$ such that $v \in k_{2}$ and $\left.v \notin T\right\}$. Consider also $T_{m}^{*}=\left\{T \cap m_{2} \mid T \in T^{*}\right\}$ for $m<\omega$. $T^{*}$ can be viewed as a tree if we identify it with $\bigcup_{m<\omega} T_{m}^{*}$ and define an order by setting $t_{1} \triangleleft t_{2}$ iff for some $m<\omega t_{1}=t_{2} \cap^{m>2}$. Then, clearly,

$$
\|_{Q_{1}}^{\prime \prime} \tau \text { is an } \omega-\text { branch of } T^{* \prime \prime} .
$$

Claim 3.5. Suppose that $n<\omega, q_{0} \in$ Random, $\eta \in{ }^{n} 2$. Then there are $m<\omega$, $q, v_{0}, v_{1}, t_{0}, t_{1}$ such that

(a) $q \in$ Random and $q \geq q_{0}$.

(b) $\eta \triangleleft v_{0}, v_{1} \in \omega>2$

(c) $t_{0}, t_{1} \subseteq m \geq 2$ and $t_{0} \neq t_{1}$.

(d) $\left(q, v_{i}\right) \Vdash \underset{\sim}{\tau} \cap^{m \geq 2}=t_{i}$ for $i<2$. 
Proof: Find first some $q^{\prime} \geq q_{0}$ and $v_{0} \triangleleft \eta$ deciding $\underset{\sim}{\tau} \cap^{m \geq} \geq_{2}$. Let $t_{0}$ be the decided value, i.e. $\left(q^{\prime}, v_{0}\right) \Vdash \underset{\sim}{\tau} \cap^{m \geq 2}=t_{0}$. By the Claim 3.4 there will be $m<\omega$ and $v \triangleleft \eta$, $v \in m_{2} \backslash t_{0}$. Find some $\left(q, v_{1}\right) \geq\left(q^{\prime}, v\right)$ deciding $\underset{\sim}{\tau} \cap^{m_{2}}$. Let $t_{1}$ be the forced value, i.e. $\left(q, v_{1}\right) \Vdash \underset{\sim}{\tau} \cap^{m} 2=t_{1}$. Since $\left(q, v_{1}\right) \Vdash v_{1} \in \underset{\sim}{\tau}$, we have $\left(q, v_{1}\right) \Vdash v=v_{1}\lceil m \in \underset{\sim}{\tau}$. But this means $t_{o} \neq t_{1}$.

Claim 3.6. Suppose that $n, k<\omega$ and $q_{0} \in$ Random. Then there are $q \in$ Random, $m<\omega,\left\langle v_{\eta, \ell} \mid \eta \in{ }^{n} 2, \ell<k\right\rangle$ and $\left\langle t_{\eta, \ell} \mid \eta \in{ }^{n} 2, \ell<k\right\rangle$ such that
(a) $q \geq q_{0}$
(b) $m \geq n$
(c) for every $\eta_{1}, \eta_{2} \in{ }^{n_{2}}, \ell_{1}, \ell_{2}<k t_{\eta_{1}, \ell_{1}}=t_{\eta_{2}, \ell_{2}}$ iff $\left(\eta_{1}, \ell_{1}\right)=\left(\eta_{2}, \ell_{2}\right)$
(d) for every $\eta \in{ }^{n} 2, \ell<k$

$$
\eta \triangleleft v_{\eta, \ell} \in{ }^{\omega>} 2, t_{\eta, \ell} \in T_{m}^{*} \text { and }\left(q, v_{\eta, \ell}\right) \Vdash \underset{\sim}{\sim} \cap^{m_{2}}=t_{\eta, \ell} .
$$

Proof: Just use the previous claim enough times. Thus, first, we generate a tree of $k \cdot\left(2^{n}+1\right)$ possibilities for one $\eta \in n^{n}$ and then we repeat the argument of Claim 3.5 on all $\eta$ 's.

Claim 3.7. For every $n<\omega, k<\omega, q^{\prime} \in$ Random and $\mathcal{E}>0$ there are $m<\omega$, $q \geq q^{\prime},\left\{q_{\ell} \mid \ell<\ell^{*}\right\} \subseteq$ Random pairwise disjoint, $\left\langle v_{\eta, \ell, j} \mid \eta \in{ }^{n} 2, \ell<\ell^{*}, j<k\right\rangle$ and $\left\langle t_{\eta, \ell, j} \mid \eta \in{ }^{n} 2, \ell<\ell^{*}, j<k\right\rangle$ such that

(a) $L b(q) \geq 1-\mathcal{E}(L b$ denotes the Lebesgue measure)

(b) $q=\bigcup_{\ell<\ell^{*}} q_{\ell}$

(c) if $\eta \in{ }^{n} 2, \ell<\ell^{*}$ and $j<k$ then $v_{\eta, \ell, j} \in{ }^{\omega>} 2 v_{\eta, \ell, j} \triangleright \eta$ and $\left(q_{\ell}, v_{\eta, \ell, j}\right) \Vdash \underset{\sim}{\tau} \cap^{m \geq} \geq_{2}=t_{\eta, \ell, j}$.

(d) for every $\ell<\ell^{*}$

$$
t_{\eta_{1} \ell, j_{1}}=t_{\eta_{2}, \ell, j_{2}} \quad \text { iff } \quad\left(\eta_{1}, j_{2}\right)=\left(\eta_{2}, j_{2}\right)
$$

Proof: We define by induction qe's using Claim 3.6. Thus if $\left\langle q_{i} \mid i \leq \ell\right\rangle$ is defined then we apply Claim 3.6 to ${ }^{\omega} \backslash \backslash \bigcup_{i \leq \ell} q_{i}$. The process stops after we reach $\ell^{*}$ s.t. $L b\left(\bigcup_{\ell<\ell^{*}} q_{\ell}\right) \geq 1-\mathcal{E}$. 
Claim 3.8. For every $n<\omega$ and $\mathcal{E}>0$ there are $m, n \leq m<\omega$ and a function $H: T_{m}^{*} \longrightarrow 2$ such that for every $\eta \in{ }^{n} 2$ and $i \in 2$ we can find $q^{i, \eta}, \ell^{i, \eta}<\omega,\left\langle q_{\ell}^{i, \eta} \mid \ell<\ell^{i, \eta}\right\rangle$ and $\left\langle v_{\ell}^{i, \eta} \mid \ell<\ell^{i, \eta}\right\rangle$ such that for every $i<2$ and $\ell<\ell^{i, \eta}$

(a) $\eta \triangleleft v_{\ell}^{i, \eta} \in \omega>2$

(b) $q^{i, \eta}, q_{\ell}^{i, \eta} \in$ Random and $q^{i, \eta}=\bigcup_{\ell<\ell^{i}} q_{\ell}^{i, \eta}$

(c) $L b\left(q^{i, \eta}\right) \geq 1-\mathcal{E}$

(d) $\left\langle q_{\ell}^{i, \eta} \mid \ell<\ell^{i, \eta}\right\rangle$ are pairwise disjoint

(e) $\left(q_{\ell}^{i, \eta}, v_{\ell}^{i, \eta}\right) \Vdash$ " $H\left(\underset{\sim}{\tau} \cap^{m \geq 2}\right)=i "$.

Proof: For every $\eta \in{ }^{n} 2$ and $t \in T_{m}^{*}$ let $I_{\eta, t}=\left\{q \in\right.$ Random | there is $v \in{ }^{\omega>2} 2, v \triangleleft \eta$ such that $\left.(q, v) \Vdash{ }^{\prime \prime} \underset{\sim}{\sim} \cap^{m \geq 2}=t^{\prime \prime}\right\}$. Let $\left\{q_{\eta, t, \ell} \mid \ell<\ell_{\eta, t} \leq \omega\right\}$ be a maximal antichain subset of $I_{\eta, t}$. Let $q_{\eta, t}^{*}=\bigcup_{\ell<\ell_{\eta, t}} q_{\eta, t, \ell}$. Then $\bigcup_{t \in T_{m}^{*}} q_{\eta, t}^{*}={ }^{\omega} 2 \bmod$ null set, since $\left\{q_{\eta, t, \ell} \mid t \in T_{m}^{*}\right.$, $\left.\ell<\ell_{\eta, t}\right\}$ is a predense subset of Random (but not necessarily antichain). So $L b\left(\bigcup_{t \in T_{m}^{*}} q_{\eta, t}^{*}\right)=$ 1.

It is enough to prove the following statement:

$\left.{ }^{*}\right)$ There exists $H: T_{m}^{*} \longrightarrow 2$ so that for every $\eta \in{ }^{n} 2$ and $i<2$

$$
L b\left(\bigcup\left\{q_{\eta, t}^{*} \mid t \in T_{m}^{*} \text { and } H(t)=i\right\}\right) \geq 1-\frac{\mathcal{E}}{2} .
$$

Since then we will be able to find a maximal antichain $\left\langle q_{\ell}^{i} \mid \ell<\ell^{*} \leq \omega\right\rangle$ in Random above $\bigcup\left\{q_{\eta, t}^{*} \mid t \in T_{m}^{*}\right.$ and $\left.H(t)=i\right\}$ together with $\left\langle v_{\ell}^{i} \mid \ell<\ell^{*}\right\rangle$ and $\left\langle t_{\ell}^{i} \mid \ell<\ell^{*}\right\rangle$ so that

$$
\left(q_{\ell}^{i}, v_{\ell}^{i}\right) \Perp_{\sim}^{\prime \prime} \cap^{m \geq_{2}}=t_{\ell}^{i} \text { and } H\left(t_{\ell}\right)=i^{\prime \prime} .
$$

In order to reduce $\ell^{*}$ to a finite $\ell^{i}$ we note that the precision here is $1-\frac{\mathcal{E}}{2}$ but only $1-\mathcal{E}$ is needed.

So let us prove $\left({ }^{*}\right)$. We consider the set $\mathcal{H}$ of all functions $H: T_{m}^{*} \longrightarrow 2$. It is finite but more transparent is to look at it as a probability space. All $H \in \mathcal{H}$ with the same probability. So we choose $H(t) \in\{0,1\}$ independently for the $t \in T_{m}^{*}$ with probability $1 / 2$.

We use $m$ given by Claim 3.7 for our $n, \mathcal{E}^{\prime}$ much smaller than $\mathcal{E}$ and $k$ large enough. Given $\eta \in{ }^{n} 2$ and $i<2$. We consider the probability of

$$
\left(L b\left(\bigcup\left\{q_{\eta, t}^{*} \mid t \in T_{m}^{*}, H(t)=i\right\}\right) \geq 1-\frac{\mathcal{E}}{2}\right)
$$


in $\mathcal{H}$. It is $\leq 1$, as the value is always $\leq 1$ and is $\geq 1-\frac{1}{2^{k}}$. In order to prove the last inequality, let us use $\left\{q_{\ell} \mid \ell<\ell^{*}\right\}$ of Claim 3.7. Thus

$$
\begin{aligned}
& L b\left(\bigcup\left\{q_{\eta, t}^{*} \mid t \in T_{m}^{*} \text { and } H(t)=i\right\}\right. \\
& =\sum_{\ell<\ell^{*}} L b\left(q_{\ell} \cap \bigcup\left\{q_{\eta, t}^{*} \mid t \in T_{m}^{*} \text { and } H(t)=i\right\}\right)+L b\left[\left(\omega_{2} \backslash \bigcup_{\ell<\ell^{*}} q_{\ell}\right) \cap\right. \\
& \left.\left(\bigcup\left\{q_{\eta, t}^{*} \mid t \in T_{m}^{*} \text { and } H(t)=i\right\}\right)\right] \geq \\
& \geq \sum_{\ell<\ell^{*}}\left[L b\left(q_{\ell}\right) \times\left(L b\left(q_{\ell} \cap \bigcup\left\{q_{\eta, t}^{*} \mid t \in T_{m}^{*} \text { and } H(t)=i\right\}\right)\right] / L b\left(q_{\ell}\right)-\mathcal{E}^{\prime}\right.
\end{aligned}
$$

Since (a) of 3.7, $L b\left(\omega_{2} \backslash \bigcup_{\ell<\ell^{*}} q_{\ell}\right)<\mathcal{E}^{\prime}$. Now it suffices to show that for each $\ell<\ell^{*}$

$$
L b\left(q_{\ell} \cap \cup\left\{q_{\eta, t}^{*} \mid t \in T_{m}^{*}, H(t)=i\right\}\right) / L b\left(q_{\ell}\right) \geq 1-\frac{\mathcal{E}}{4}
$$

holds for enough $H$ 's. But $v_{\eta, \ell, j}, t_{\eta, \ell, j}(j<k)$ of 3.7 are witnessing that the probability in $\mathcal{H}$ of the failure is $\leq \frac{1}{2^{k}}$. Just in order to fail, $H$ should take the value $1-i$ on $t_{\eta, \ell, j}$ for every $j<k$ and the probability of 0,1 are equal. The probability of the failure for some $\eta \in{ }^{n}, i \in 2$ is then $\leq \frac{2^{n+1}}{2^{k}}$. So, picking $k$ large enough comparatively to $n$ we will insure that most $H \in \mathcal{H}$ are fine, whereas we need only one.

Now using Claim 3.8, we define by induction on $j<\omega n_{j}, m_{j}, H_{j},\left\langle q_{j}^{i, \eta} \mid i<2, \eta \in^{n_{j}} 2\right\rangle$ $\left\langle q_{j, \ell}^{i, \eta} \mid \ell<\ell_{j} i<2, \eta \in{ }^{n j} 2\right\rangle$ and $\left\langle v_{j, \ell}^{i, \eta} \mid \ell<\ell_{j}, i<2, \eta \in{ }^{n_{j}} 2\right\rangle$ such that

(1) $n_{j}<m_{j}<n_{j+1}$

(2) $m_{j} H_{j},\left\langle q_{j}^{i, \eta} \mid i<2, \eta \in{ }^{n_{j}} 2\right\rangle,\left\langle q_{j, \ell}^{i, \eta} \mid \ell<\ell_{j}^{\eta, i}, i<2, \eta \in{ }^{n_{j}} 2\right\rangle$ and $\left\langle v_{j, \ell}^{i, \eta}\right| \ell<\ell_{j}^{i, \eta}, i<$ $\left.2, \eta \in{ }^{n_{j}} 2\right\rangle$ are given by Claim 3.8 for $n=n_{j}$ and $\mathcal{E}=\frac{1}{2^{2^{n_{j}}}}$

(3) length $\left(v_{j, \ell}^{i, \eta}\right)<n_{j+1}$ for every $i<2, \eta \in{ }^{n_{j}} 2, \ell<\ell_{j}^{i, \eta}$.

Now define a $Q_{1}$-name $\underset{\sim}{\sigma \in}{ }^{\omega_{2}}$ by setting

$$
\underset{\sim}{\sigma}(j)=H_{j}\left(\underset{\sim}{\tau} \cap^{m_{j} \geq 2}\right)
$$


Claim 3.9. $\Vdash{ }_{Q}$ " $\sigma$ is a Cohen real over $V "$.

Proof: It is enough to show the following:

$\circ$ for every $\mathcal{E}>0$ and $\eta^{*} \in{ }^{\omega>}>2$ the following holds:

for every $j<\omega$ large enough and $\nu \in{ }^{\omega>} 2$ of the length $>j$ there are $q,\left\langle q_{\ell} \mid \ell<\ell^{*}\right\rangle$ and $\left\langle v_{\ell} \mid \ell<\ell^{*}\right\rangle$ such that

(a) $q,\left\langle q_{\ell} \mid \ell<\ell^{*}\right\rangle$ are in Random

(b) $q=\bigcup_{\ell<\ell^{*}} q_{\ell}$

(c) $\operatorname{Lb}(q) \geq 1-\mathcal{E}$

(d) $v_{\ell} \unlhd \eta^{*}$ for every $\ell<\ell^{*}$

(e) $\left(q_{\ell}, v_{\ell}\right) \Vdash \longmapsto_{Q}^{\prime \prime} \underset{\sim}{\sigma} \uparrow[j$, length $\nu)=\nu \uparrow[j \text {, length } \nu)^{\prime \prime}$.

Proof of o. Pick $j<\omega$ such that $n_{j}>$ length $\eta^{*}$ and $2^{-j}<\frac{\mathcal{E}}{2}$. Let $\nu$ be given. We choose by induction on $k \in\left[j\right.$, length $\nu$ ) a set $a_{k}$ and $\left\langle q_{\eta} \mid \eta \in a_{k}\right\rangle$ such that

(a) $a_{k} \subseteq{ }^{n_{k}} 2$ is nonempty.

(b) $a_{j}$ is a singleton extending $\eta^{*}$

(c) $\forall \eta \in a_{k+1}\left(\eta\left\lceil n_{k} \in a_{k}\right)\right.$ and $\forall \eta \in a_{k} \exists \eta^{\prime} \in a_{k+1}\left(\eta \triangleleft \eta^{\prime}\right)$.

(d) for every $\eta \in a_{k}$

$$
\left(q_{\eta}, \eta\right) \Vdash \bigwedge_{\ell=j}^{\prime \prime} H_{\ell}\left(\underset{\sim}{\tau} \cap^{m_{\ell} \geq_{2}}\right)=\nu(\ell)^{\prime \prime}
$$

i.e. $\left(q_{\eta}, \eta\right) \Vdash{ }^{\prime \prime} \underset{\sim}{\sigma} \uparrow[j, k-1)=\nu \uparrow[j, k-1)^{\prime \prime}$

(e) for $\eta \in a_{j} q_{\eta}=\omega_{2}$

(f) for $\eta \in a_{k}\left\langle q_{\rho} \mid \eta \triangleleft \rho \in a_{k+1}\right\rangle$ is an antichain of Random above $q_{\eta}$ and $\sum\left\{L b\left(q_{\rho}\right) \mid\right.$ $\left.\eta \triangleleft \rho \in a_{k+1}\right\} / L b\left(q_{\eta}\right) \geq 1-\frac{1}{2^{k}}$.

There is no problem in caring on this induction. This completes the proof of $\circ$ and hence also the theorem. 


\section{References}

[Fr] D. Fremlin, Real-valued measurable cardinals in Set Theory of the Reals H. Judah ed., Israel Math. Conf. Proceedings (1993), 151-305.

[Fr2] D. Fremlin, Measure Algebras, in J.D. Monk ed., Handbook of Boolean Algebras, North-Holland (1989), 876-980.

[Gi-Sh1] M. Gitik and S. Shelah, On simple forcing notions and forcing with ideals, Israel J. Math. 68(2) (1989) 129-160.

[Gi-Sh2] M. Gitik and S. Shelah, More on simple forcing notions and forcing with ideals Ann. of Pure and Appl. Logic 59 (1993), 219-238.

[Pr] K. Prikry, Ideals and powers of cardinals, Bull. AMS 81 (1975), 907-909.

[So] R. Solovay, Real-valued measurable cardinals, in: D. Scott, ed. Axiomatic Set Theory, Proc. Symp. Pure Math. 13(1), (1970), 397-428.

[Sh1] S. Shelah, Cardinal and Arithmetic, Oxford Logic Guides 29, Oxford Science Publications 1994.

[Sh430] S. Shelah, Further Cardinal Arithmetic, [Sh430]

[Sh460] S. Shelah, The Generalized Continuum Hypothesis revisited, [Sh460], to appear.

[Sh580] S. Shelah, On Strong Covering, [Sh580]. 